\title{
A NEW WINDOW COATING SYSTEM AND A NEW WINDOW FOR THE ALS*
}

\author{
$\underline{\text { C.C. Lo }}^{* *}$, J. Julian, K. Baptiste and B. Taylor ${ }^{* * *}$ \\ Advanced Light Source \\ Lawrence Berkeley National Laboratory \\ 1 Cyclotron Road, \\ Berkeley, California USA 94720
}

\begin{abstract}
A new window coating system has been developed to coat a newly designed window for the Storage Ring RF System in the Advanced Light Source(ALS). A bell jar large enough to accommodate the $15 \mathrm{~cm}(\mathrm{OD}) \times 50 \mathrm{~cm}$ (L) tubular ceramic window is used for a vacuum chamber. Methodical procedures are employed to ensure the chamber is free of contaminants before any coating is carried out. A single filament is used to coat the new tubular window, however other shapes of filaments could be used for coating other windows if necessary. Details of the coating system and window construction as well as the performance of the coating system and the new windows will be presented.
\end{abstract}

\section{INTRODUCTION}

In the ALS Booster and Storage ring the RF cavities operate at ultra high vacuum (UHV) and require ceramic windows to serve as barriers between UHV and atmospheric pressure. These windows enable the cavity to be isolated and at the same time allows Radio Frequency (RF) signals to be transmitted from the wave guide to the cavity. Since these windows are installed at the window coupling ports of the cavities they must be able to withstand the high electric field of the cavity, have low RF power dissipation and present a low secondary electron emission coefficient to reduce multipactoring that usually leads to catastrophic break downs. The last item dictates that the ceramic window be coated with materials which would provide a small secondary electron emission coefficient. Another very important function of the coating is to drain the charge on the ceramic away before it is charged to a flash over potential. Therefore it is imperative that the two ends of the ceramic tube be metalized around the perimeter at the ends and $1 \mathrm{~mm}$ into the inner wall so that the coating can make connection to ground through them.

\section{THE COATING SYSTEM}

Fig. 1 is a diagram showing the details of the coating system. The bell jar has a diameter of $48 \mathrm{~cm}$ and a height of $76 \mathrm{~cm}$. The total height of the tubular window including flanges measures $53.2 \mathrm{~cm}$. The titanium filamentruns straight through from the top to the

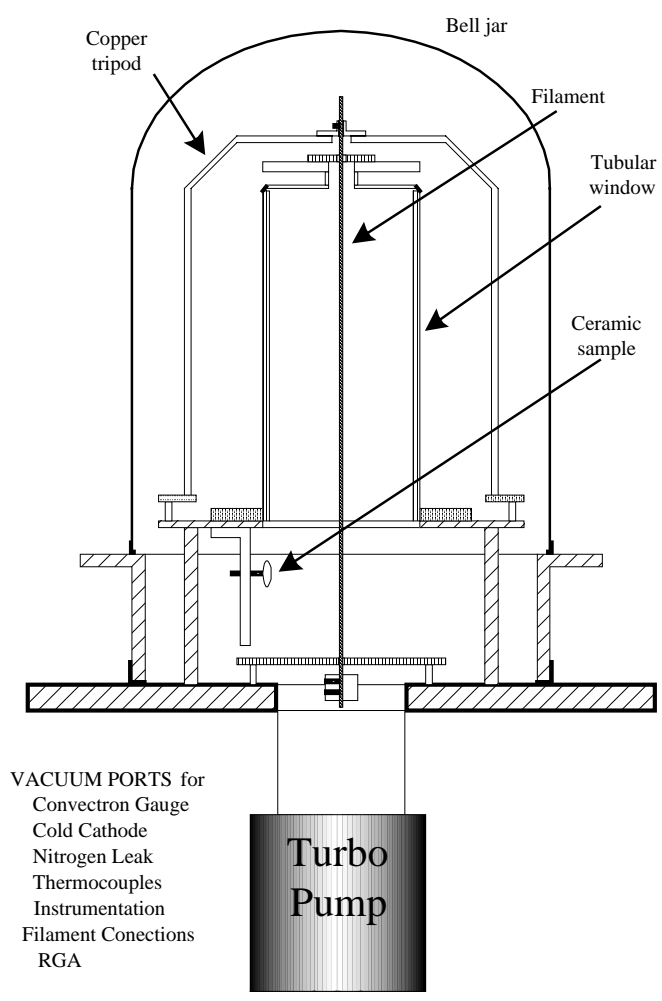

Fig. 1 Coating System

bottom of entire length of the window and beyond. It is important to have the window's inside surface facing a uniformly heated portion of the filament as much as possible to obtain a uniform coating. Power is delivered to the top connection of the filament via a tripod supporting structure. The lower part of the filament is weighted down to keep the filament straight through out the coating cycle. A turbo pump is used in series with a dry scroll pump to evacuate the bell jar chamber. A residual gas analyzer (RGA) is used to look at the evacuated chamber for any unusually high residuals before starting the coating process. After many discussions with others ${ }^{1,2,3}$ who have done this process before we decided to coat the ceramic window with a coat of approximately 10 to 20 Angstrom (1$2 \mathrm{~nm})^{4}$ of Titanium Nitride (TiN). The filament operates on 0 to $30 \mathrm{~V} \mathrm{AC}$ power. 


\section{THE WINDOW}

On going effort for aperture window development has been in progress for at least 30 years, pursuing a course from DESY to Daresbury to LBNL. The Daresbury Laboratory S.R.S. developed a series of ceramic windows that can handle a maximum of $45 \mathrm{~kW} \mathrm{cw} R F$ power into the unloaded cavity. Power was limited by ceramic charging effects resulting in non destructive flashover on the atmospheric side of the window.

The ALS requires two cavity windows rated at $70 \mathrm{~kW}$ $\mathrm{cw}$ input each and a total input power with beam of $250 \mathrm{~kW}$. A new window was designed in which the ceramic disc was replaced by a ceramic tube with a metal top cap that can be fitted to the waveguide upper wall. Typically a disc window could provide a coupling factor up to 5. Without a ceramic disc loading the cavity port, the tubular window has a lower coupling factor. By adding loading vanes at the bottom flanges as in Fig. 3, the cut off frequency of the aperture was lowered to the benefit of the coupling factor attainable, both for the input power and exit HOM frequencies. Typically a coupling factor of 3 can be achieved with the loading vanes. Figure 2 is a diagram of the ceramic tubular window that has a length of $50 \mathrm{~cm}$ and an internal diameter of $14 \mathrm{~cm}$. The thickness of the ceramic tube is $0.5 \mathrm{~cm}$. The bottom flange is made out of oxygen free copper with water cooling chamber and is brazed to the ceramic tube. It also has a stainless steel knife-edge that provides UHV seal to the cavity connecting flange. The top flange construction incorporates a flexible diaphragm to accommodate thermal expansion and alignment problems. The two loading vanes for obtaining higher coupling factor are integral parts of the bottom flange.

\section{THE COATING PROCESS}

The tubular ceramic RF window is given a thorough cleaning of the through and blind holes to remove all lubrication products by cleaning with acetone, isopropyl alcohol, and finally ethyl alcohol. The whole window is UHV cleaned in the plating shop, and then mounted in the vacuum coating system. Temperature probes are installed at the top, middle, and bottom of the ceramic for monitoring of the bake-out and coating temperature. A titanium filament wire, $2.0 \mathrm{~mm}$ diameter of $99.7 \%$ purity, is installed through the center of the tubular window. A vertical filament wire is used twice before being replaced, and the lower ring filament wire is being used only once because of mounting difficulties, sagging, and becoming very brittle after a single run. This lower ring filament is used to coat the lower part of the ceramic tube in the older version of the window only. The new windows do not need this extra coating process. Verification targets are installed to verify the thickness of the TiN coating of every run. The vacuum coating system base, spool, and bell jar are wiped down with ethyl alcohol

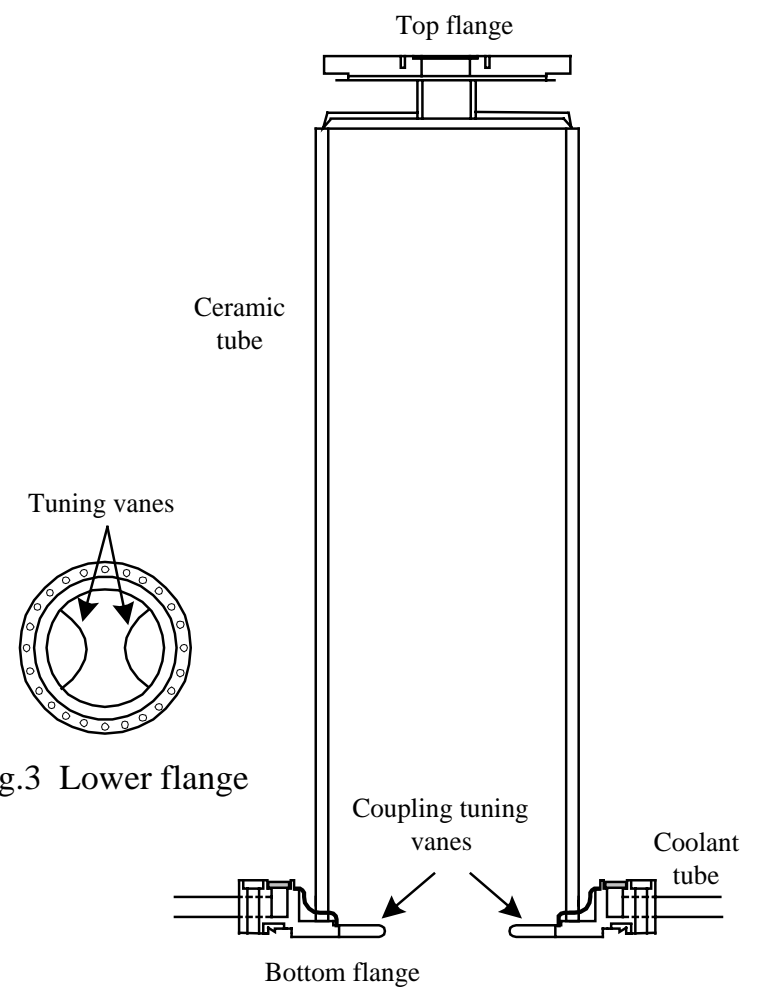

Fig. 2 RF Window

and the vacuum system is closed up for pumping. A Varian 600DS-scroll vacuum pump with a turbo-V $300 \mathrm{HT}$ pump is used to pump down the chamber. Four 250W IR lamps, a base plate heater that can provide a maximum temperature of $70^{\circ} \mathrm{C}$ and the $\mathrm{Ti}$ filament running between $10 \mathrm{~A}$ to $13 \mathrm{~A}$ are used to bake out the vacuum chamber. This low temperature bake will last for a period of 3 to 5 days or until the vacuum pressure is in the low $10^{-7}$ Torr range or better. An RGA scan is taken to check for water, petroleum products, etc. before coating.

Two hours before the coating run, the cold trap is filled and the nitrogen lines are purged for twenty minutes from a full Dewar flask. Next, the vacuum chamber is purged with dry nitrogen at a pressure of 3 to $8 \times 10^{-4}$ Torr via the .01 micron particle filter through the leak valve for period of ten to twenty minutes. This nitrogen purge is repeated for a total of up to four times. The optical pyrometer and Infrared thermometer are mounted on fixed supports and aligned using an illumination lamp. The two units are used to monitor the filament wire at the base of the window. Ten minutes before coating, the IR lamps and the base heater are secured, and the nitrogen leak is set for $5 \times 10^{-5}$ Torr. At the start of the coating run, the timing clock is started and the filament is brought up to 30A. After fifteen seconds, the filament current is adjusted for a filament temperature of $1050^{\circ} \mathrm{C}$. The optical pyrometer temperature is used as the reference for the coating process. The infrared thermometer is used as a backup because of its limited focal size on the wire that has a tendency to change if moved. Throughout the coating process, the filament voltage 
and the nitrogen leak are adjusted repeatedly to maintain the prescribed coating parameters. All readings are logged every ten minutes. At the completion of the 2-hour coating run, the temperature of the ceramic window will have risen from $60^{\circ} \mathrm{C}$ to $114^{\circ} \mathrm{C}$. The filament current is reduced to $10 \mathrm{~A}$ for a period of one hour for cool down, and the nitrogen pressure in the bell jar is increase to $5 \times 10^{-4}$. At the end of one hour, the filament is turned off, bell jar isolation valve is closed, vacuum pumps secured, and then the bell jar is back filled with nitrogen pressure to $1 \times 10^{1}$ Torr.

\section{WINDOW PERFORMANCE}

We have used the coating system to coat four tubular windows. The coatings on all four windows were successful. Of the four windows two were new ones made for us by EEV of England, and the other two were reclaimed units from a different vendor. The two reclaimed windows came with some unmanageable physical flaws and hence can only be used at lower power levels and are serving as emergency backup units. We experienced an unusually high operating temperature in one of the two new windows since conditioning and the temperature has not come down as yet. The cause of the high operating temperature is not entirely clear at this time however it could be due to the fact that this window was rejected by the factory due to a vacuum leak and subsequently was not handled or cleaned as a new window. The vacuum leak, however, was circumvented in LBNL after many iterations of solution. The plan is to remove this window at a convenient time, have it cleaned, alumina bead blasted and re-coat. Despite its higher than normal operating temperature (above $100^{\circ} \mathrm{C}$ ) this window is free of voltage breakdowns. The fourth window operates without any problem and both new windows were conditioned up to full power within a relatively short time (a few hours).

\section{CONCLUSION}

Our new coating system performs very well. The recipe we have developed for coating this type of windows is quite effective and the performance of the coating is consistent. We are still in the process of acquiring a few more good windows for ALS.

\section{REFERENCES}

1. Rich Callen, Private communication. SLAC

2. Karen Cummings, Private communication. LANL

3. K. Primdahl, R. Kustom, J. Maj

"Reduction of Multipactor in RF Ceramic Windows Using a Simple Titanium-Vapor Deposition System", IEEE PAC Proceedings, May 1-5,1995, pp 1687-1689.

4. Y. Saito, "Breakdown Phenomena in Vacuum”, KEK. Linear Accelerator Conference Proceedings (1992)

*Work supported by the Director, Office of Energy Research, Office of Basic Energy Science, Materials Science Division, of the U.S. Department of Energy under Contract No. DE-AC03-76F00098

\footnotetext{
**cclo@lbl.gov

$* * *$ Retired
} 